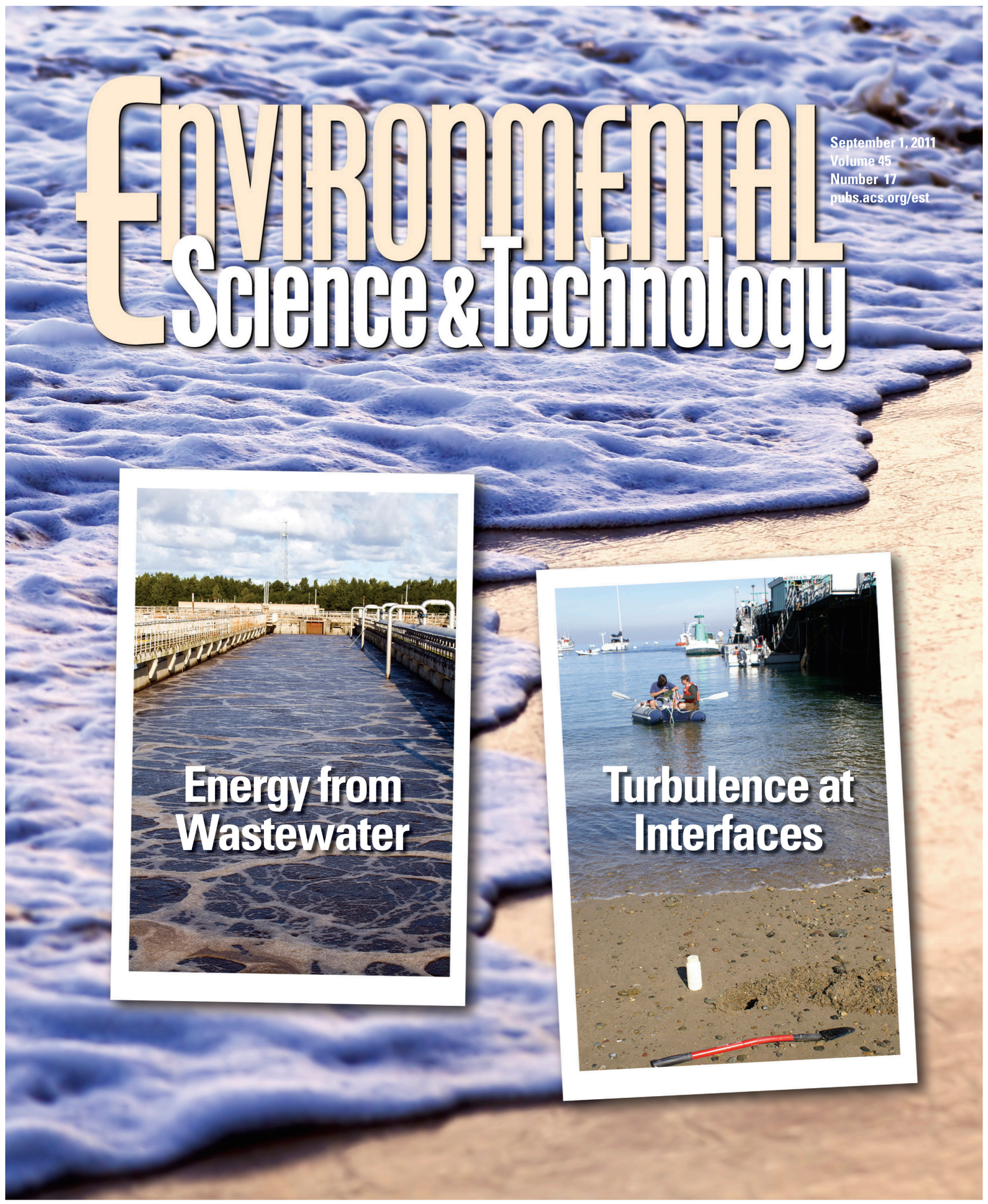

ACS Publications

www.acs.org 


\title{
Crossing Turbulent Boundaries: Interfacial Flux in Environmental Flows
}

\author{
Stanley B. Grant ${ }^{*,+, \neq}$ and Ivan Marusic ${ }^{\S}$ \\ ${ }^{\dagger}$ Department of Civil and Environmental Engineering, Henry Samueli School of Engineering, University of California, Irvine, \\ California 92697, United States \\ ${ }^{\ddagger}$ Department of Infrastructure Engineering and ${ }^{\S}$ Department of Mechanical Engineering, Melbourne School of Engineering, \\ University of Melbourne, Victoria 3010, Australia
}

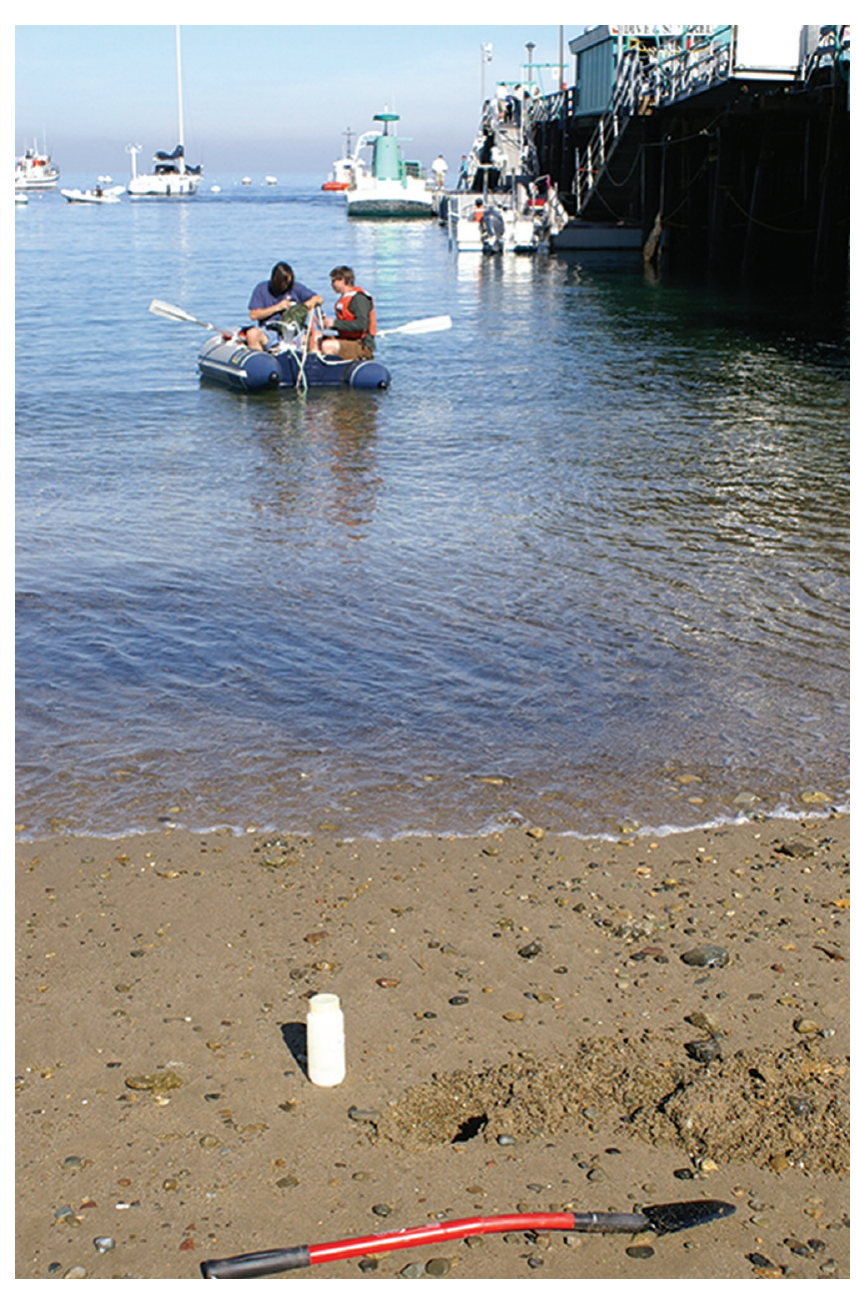

Tany processes of interest to environmental scientists and 1 engineers are influenced by turbulent fluid motion at an interface. ${ }^{1,2}$ Textbooks often assume interfacial turbulence is isotropic and homogeneous, but flow visualization studies have long demonstrated the existence of organized "coherent structures" in the turbulence field that violate both assumptions. ${ }^{3-7}$ Coherent turbulent structures spawn highly chaotic "sweep and eject events" in which high velocity fluid sweeps down into the free stream turbulent boundary layer, and low velocity fluid at the interface is ejected into the bulk flow. The future challenge is to develop new theories and practical approaches for estimating interfacial transport rates that properly account for these coherent turbulent structures. The goal of this article is to provide an overview of recent advances in the study and characterization of turbulence at interfaces, and their implications for environmental science and engineering.

\section{CANONICAL MODEL OF THE TURBULENT BOUND- ARY LAYER}

The canonical model of turbulent boundary layers can be traced to Ludwig Prandtl who, in the early 1900s, discovered that the drag force on a submerged object in a flowing fluid can be calculated by dividing the flow field into two regions - a turbulent velocity boundary layer close to the surface where viscous forces are important, and a region outside of the turbulent boundary layer where viscous forces are negligible. ${ }^{8}$ Careful measurements of the mean velocity profile in turbulent boundary layers, carried out in the 1930 s and later ${ }^{9,10}$ revealed that the turbulent boundary layer can be further divided into inner and outer regions, each characterized by a unique set of scaling variables (Figure 1A and Box 1).

1: INNER AND OUTER REGION OF THE TURBULENT BOUNDARY LAYER: Inner Region. In the inner region of the turbulent boundary layer, the mean velocity profile, $(U(y))$ and turbulence statistics are presumed to depend only on the distance from the wall $(y)$, the kinematic viscosity of the fluid $\left(v=\mu / \rho\right.$, units $\mathrm{m}^{2} \mathrm{~s}^{-1}$, where $\mu$ and $\rho$ represent the dynamic viscosity and density of the fluid, respectively) and the shear stress exerted on the boundary as characterized by the shear velocity $\left(u_{*}=\left(\tau_{\mathrm{w}} / \rho\right)^{1 / 2}\right.$ with units $\mathrm{m} \mathrm{s}^{-1}$, and $\tau_{\mathrm{w}}$ represents the wall shear stress):

$$
\frac{U_{\text {inner }}}{u *}=f\left(\frac{y u *}{v}\right) \text { or } U_{\text {inner }}^{+}=f\left(y^{+}\right)
$$

The nondimensional variables in the second equation are $U_{\text {inner }}^{+} \equiv U_{\text {inner }} / u_{*}$ and $y^{+} \equiv y u^{*} / v$. The inner region includes: (1) the viscous sublayer $\left(0 \leq y^{+} \leq 5\right)$ where turbulent fluctuations are suppressed, molecular viscosity dominates momentum transport, and the mean velocity increases linearly with distance from the wall $\left(U_{\text {inner }}^{+}=y^{+}\right)$,

Published: July 27, 2011 
(2) a fully turbulent region, which overlaps with the outer region, where momentum transport is dominated by turbulent fluctuations or, more precisely, the Reynolds stresses, and (3) a buffer region transitional between (1) and (2). The inner region extends from the wall to a distance of approximately $y=0.15 \delta$, where $\delta$ is the thickness of the velocity boundary layer, defined by the location where the flow velocity is $99 \%$ of the free stream velocity, $U(\delta)=0.99 U_{\infty}$.

Outer Region. In the outer region, the turbulence statistics and the mean velocity profile (which is characterized by the velocity deficit $U(y)-U_{\infty}$ where $U_{\infty}$ is the free-stream velocity) depend on the shear velocity $u_{*}$ and the boundary layer thickness $\delta$, but not on kinematic viscosity (because momentum transport is entirely dominated by Reynolds stresses):

$$
\frac{U_{\infty}-U}{u *}=g\left(\frac{y}{\delta}\right)
$$

The outer region extends from nominally $30 / \operatorname{Re}_{\tau}<y / \delta<1$ where $\operatorname{Re}_{\tau} \equiv \delta u_{*} / v$ represents the ratio of the outer $(\delta)$ and inner $\left(v / u_{*}\right)$ region length-scales.

Overlap Region and "Law of the Wall". Where the inner and outer regions overlap, eqs 1 and 2 imply the existence of a logarithmic relationship between the mean velocity and distance from the wall (Figure 1A):

$$
\begin{aligned}
& U^{+}=\frac{1}{\kappa} \ln y^{+}+A, \text { Prandtl's Law of the Wall } \\
& \frac{U_{\infty}-U}{u *}=-\frac{1}{\kappa} \ln \frac{y}{\delta}+c, \text { Velocity Defect Law }
\end{aligned}
$$

The von Karman constant $\kappa \approx 0.41$ and $A \approx 5$ are assumed universal (i.e., apply for all flow geometries), while the value of $c$ depends on the details of the flow field $(c \approx 2.3$ for zero pressure gradient flows). Equations $3 \mathrm{a}$ and $3 \mathrm{~b}$ are valid from $30<y^{+}<0.15 \operatorname{Re}_{\tau}$.

\section{COHERENT TURBULENCE}

Many treatments of small-scales in boundary layer turbulence assume the turbulence to be close to isotropic (i.e., statistically similar in all directions) and homogeneous (statistically similar from moment-to-moment and point-to-point) (Figure 1B), and the inner and outer regions are assumed to have no interaction. However, boundary layer turbulence deviates significantly from this idealization, due to the existence of coherent turbulent eddying motions or structures over which positive and/or negative velocity fluctuations are spatially correlated (Figure 1C). Coherent turbulence occurs over a hierarchy of spatial scales. Close to the wall it takes the form of Quasi-Streamwise Vortices (QSV) oriented parallel to the flow and extending from the wall to the buffer layer, where they are responsible for most of the turbulence kinetic energy production. At larger scales, the QSV may evolve into horseshoe shaped structures called "hairpin vortices" (Figure 1D) that extend from the wall into the logarithmic layer, ${ }^{3}$ and "super structures" characterized by regions of elongated negative velocity fluctuation (with instantaneous reported lengths of $15-20 \delta$ ) flanked by regions of positive fluctuations. ${ }^{11}$ Hairpin-type vortices and
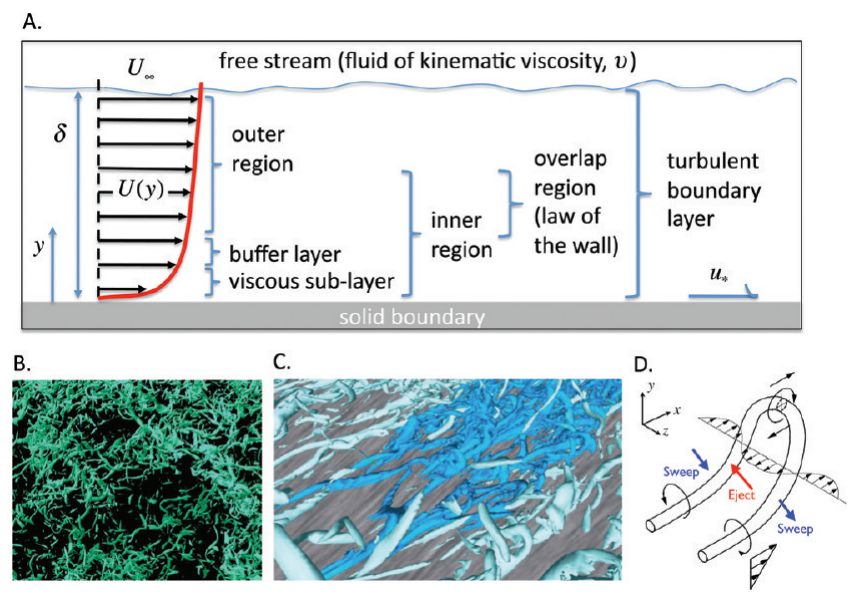

Figure 1. (A) Classical regions used to describe wall-bounded turbulence. In this description, the viscous sublayer and buffer layers are influenced by viscosity, while the outer region is not. Equation 1 holds for the inner region, while eq 2 holds for the fully turbulent outer region. Equations $3 \mathrm{a}$ and $3 \mathrm{~b}$ are assumed to hold in the overlap of the inner and outer regions, known as the (logarithmic) law of wall region. (B) A visualization of the turbulent vortices characteristic of homogeneous isotropic turbulence, based on a direct numerical simulation of grid turbulence in ref 50 . The filamentary vortices generated by the homogeneous isotropic turbulence (which are visualized as isosurfaces of mean-square vorticity) are randomly oriented and intermittent in space. Note that there is no boundary present in this figure. (C) Flow visualization of coherent eddying motions rising off a wall in turbulent flow, taken from ref 51 . Here the filamentary vortices are organized into highly anisotropic "coherent" structures, such as hairpin vortices. The boundary is represented by the gray surface. (D) An idealized hairpin-shaped vortex in wall bounded turbulence, with its induced velocity field producing "sweep" and "eject" events from and to the wall, respectively. Note that $y$ is the distance away from the wall and the arms of the hairpin vortex typically extend down in the viscous buffer layer. See Box 1 for definition of terms.

superstructures are observed in the turbulent boundary layers of pipes, channels, and flat plates, as well as in the atmospheric boundary layer where they can reach kilometer length scales. ${ }^{12,13}$

Although the precise details and form of these eddying motions are still open to debate, ${ }^{14-16}$ Figure 1D illustrates how the arms of a single hairpin vortex, which rotate in opposite directions, might give rise to sweep and eject events. Between the arms of the hairpin vortex, fluid is lifted away from the boundary causing the "ejection" of slow moving fluid into the bulk flow (indicated by the negative velocity vectors in Figure 1D). Outside the arms of the hairpin vortex, fast moving fluid from the bulk flow "sweeps" down toward the boundary, giving rise to faster than average velocity near the interface (indicated by the positive velocity vectors in Figure 1D). These coherent motions are most energetic in the overlap region, where they dominate turbulent transport, but their influence extends to the inner region, where large-scale fluctuations are linearly and nonlinearly superimposed on the near-wall turbulence, in direct violation of the canonical model's premise (outlined in the last section) that the inner and outer regions of the turbulent boundary layer are independent. ${ }^{17}$

\section{TURBULENT MASS TRANSFER ACROSS THE SEDIMENT-WATER INTERFACE}

As an illustration of the concepts described above, we now focus on a single interfacial transport problem: turbulent mass 


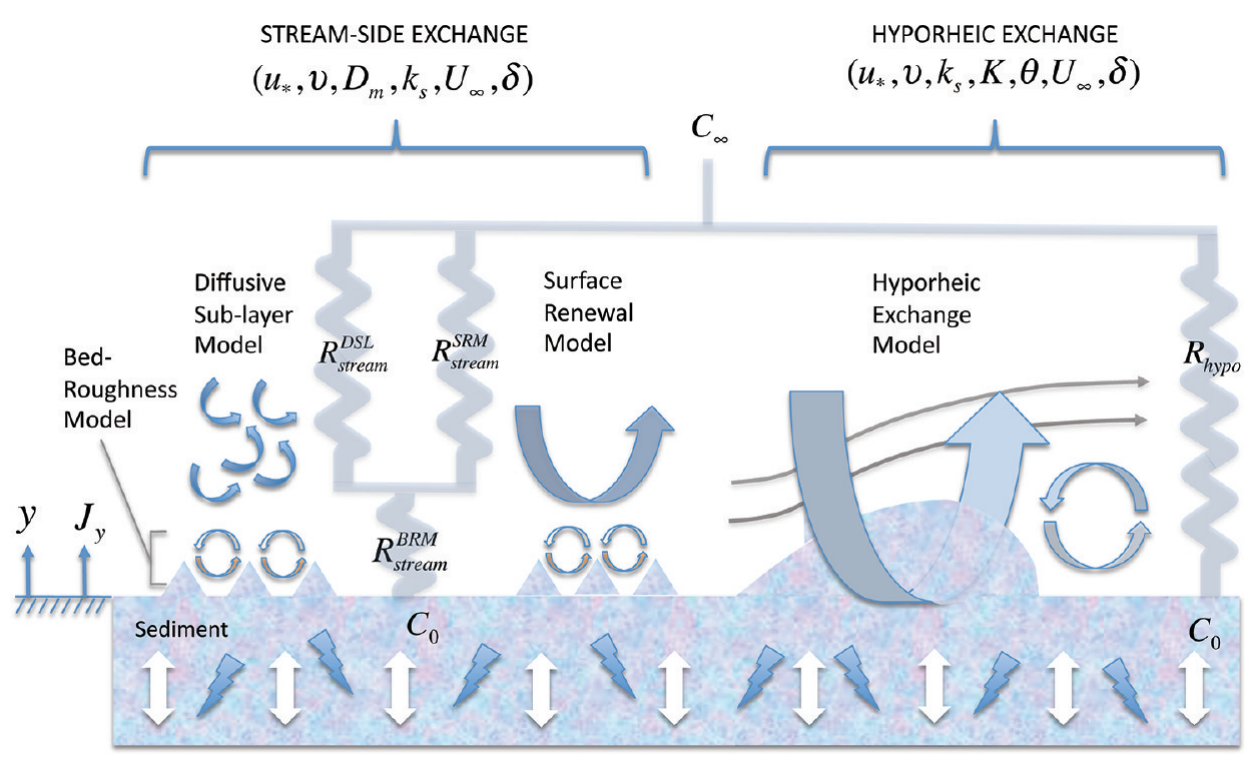

Figure 2. Processes thought to control mass transport from the bulk fluid to the surface of the sediment (streamside exchange, left) and across the sediment-water interface (hyporheic exchange, right). The arrangement of resistors in the mass transfer circuit is motivated by consideration of the length scales over which different turbulent transport processes operate, as described in the text. The bulk fluid is flowing from left to right. Double sided vertical arrows and lightening bolts represent diffusion (including biodiffusion) and reaction, respectively, in the sediment bed. The illustration for hyporheic exchange depicts detachment of the turbulent boundary layer over a large roughness element, such as a bedform or cobble. See Box 2 for definition of terms.

flux at the sediment-water interface. Turbulent flux across the sediment-water interface in rivers, lakes, and the coastal and deep ocean is integral to many biogeochemical cycles on earth, and influences the mobility and toxicity of anthropogenic contaminants and their subsequent impact on human, animal, and ecosystem health. ${ }^{18-24}$ Relevant transport processes are presented in Figure 2 and grouped into streamside exchange (left side of figure) and hyporheic exchange (right side of figure). Streamside exchange refers to the transport of mass from the bulk stream to the interface through the influence of (1) hydrodynamically controlled transport through the velocity boundary layer by homogeneous and isotropic turbulence (diffusive sublayer model $^{20}$ ) or coherent turbulence (surface renewal model ${ }^{25}$ ), and (2) turbulent transport across surface roughness elements at the sediment-water interface (bed roughness model ${ }^{20,26,27}$ ). Hyporheic exchange refers to the movement of water and mass across the interface through the influence of (1) pressure fluctuations associated with the detachment and reattachment of the turbulent boundary layer over roughness elements at the sediment-water interface such as cobbles and sediment bed forms (ripples and dunes), ${ }^{28-30}$ (2) downstream migration of sediment bed forms, ${ }^{28}$ (3) penetration of turbulent sweep and eject events into the sediment, ${ }^{31-33}(4)$ in rivers, time-averaged pressure variation along the sediment bed at the scale of riffles and pools, ${ }^{30}$ and (5) variations in sediment permeability. ${ }^{19,34}$ The microscale mechanisms illustrated in Figure 2 are embedded within a larger flow network that spans, in the case of rivers, more than a million-fold change in spatial scales, from single grains to entire catchments. ${ }^{19,30,35}$

2: RESISTANCE MODEL OF INTERFACIAL MASS

TRANSFER: The mass transfer resistance across an interval is the change in concentration $\Delta C=C_{\infty}-C_{0}$ across the interval divided by the mass flux through the interval
$J_{y}\left(\right.$ ref 36, see Figure 2 for definition of $C_{0}$ and $\left.C_{\infty}\right)$ :

$$
R=-\frac{\Delta C}{J_{y}}
$$

where the mass transfer resistance $R$ has units of inverse velocity, and is the inverse of the mass transfer coefficient frequently employed in the fields of chemical and mechanical engineering, $R=1 / k^{36}$ Equation 4 implies that, for a given value of the mass transfer resistance, the concentration change $(\Delta C)$ can be calculated from the mass flux $\left(J_{y}\right)$, or vice versa. A positive $J_{y}$ denotes mass flux in the direction of increasing $y$, where the $y$ coordinate represents elevation above the sediment bed (see coordinate system in Figure 2). The negative sign in eq 4 assures that the resistance will always be positive, because $J_{y}>0$ when $\Delta C<0$; i.e., mass flux occurs in the direction of decreasing concentration. Equation 4 is a valid description of mass transport across the SWI provided that mass flux $J_{y}$ is constant, which in turn imposes the following three conditions within the region, or "control volume", across which mass is transferred at the sediment-water interface: (1) steady-state; (2) no sources or sinks; and (3) horizontally uniform. Even if these conditions are not satisfied sensu stricto, they may be "good enough" in many cases. Few if any environmental systems are truly steady-state, but condition (1) will apply if transport rates at the interface adjust quickly to changes in the bulk flow, and thus are "quasi-steady-state" (e.g., ref 37). Condition (2) will be satisfied provided that reaction time scales are long compared to time scales associated with mass transport across the interface. ${ }^{36}$ In the case of oxygen transfer into a streambed, for example, the time scale for mass transfer is shorter than the time scale for oxygen consumption on the streamside of the interface, but transport 
and reaction occur over similar time scales on the sedimentside of the interface (e.g refs $27,38,39$ ). Condition (3) is, perhaps, the most restrictive, given that many processes of interest in turbulent environmental flows (e.g., hyporheic exchange) are characterized by considerable spatial heterogeneity. One way around this problem is to restrict consideration to mass flux that has been averaged over the spatial scale of interest, for example the "patch scale" (ca., $1-10 \mathrm{~m}$ ). This approach is consistent with current experimental methods for measuring flux across the sediment-water interface, such as recirculating flumes in the laboratory and transient dye experiments in the field, which yield patch-averaged values for relevant transport parameters. ${ }^{38,40}$ Given the substantial simplification and strong conceptual understanding afforded, the constant flux assumption is a logical starting point for an analysis of mass transport across the sediment-water interface.

Mass transfer resistance is a useful framework for conceptualizing, and potentially modeling, the transport processes illustrated in Figure 2 (Box 2). A benefit of the resistance model is that the contribution of individual transport mechanisms to the total resistance can be easily calculated for different arrangements of resistors within a mass transfer circuit. The proper arrangement of resistors in the circuit, in turn, can be deduced from an assessment of the length-scales over which specific mass transport processes operate. For example, in the case of streamside exchange it is typically assumed that mass is well mixed by turbulence through most of the water column, except in a thin $\sim 1 \mathrm{~mm}$ film (the "concentration boundary layer") immediately adjacent to the sediment-water interface where turbulent fluctuations are damped, concentration gradients form, and mass transfer is "bottlenecked" ${ }^{20}$ If hyporheic exchange is active, on the other hand, the flow of water across the sediment-water interface will presumably preclude, or sweep away, the concentration boundary layer, in which case the mass transfer bottleneck is solely hyporheic exchange. The "either/or" nature of these two transport processes is consistent with a parallel arrangement of their respective mass transfer resistors (Figure 2): ${ }^{41}$

$$
R=\left(\frac{1}{R_{\text {stream }}}+\frac{1}{R_{\text {hypo }}}\right)^{-1}
$$

Equation 5 implies that mass transport at the sediment-water interface will take the path of "least resistance", depending on the nature of turbulence above the interface $\left(u^{*}, U_{\infty}, \delta, v\right)$, biophysical properties of the sediment bed including porosity $(\theta)$ and permeability $(K)$, interfacial roughness (roughness scale $k_{s}$ ), and the molecular diffusion coefficient of the tracer $\left(D_{m}\right)$. If hyporheic exchange is negligible (e.g., because sediment hydraulic conductivity falls below the threshold of $\sim 1 \mathrm{~m}$ day $^{-1} 42$ ), then $R_{\text {hypo }} \rightarrow \infty$ and mass transport is dominated by streamside exchange, $R \approx R_{\text {stream. }}$. On the other hand, if the sediment is permeable (e.g., the hydraulic conductivity is greater than $1 \mathrm{~m} \mathrm{day}^{-1}$ ) and hyporheic exchange is active, then $R_{\text {hypo }} \ll$ $R_{\text {stream }}$ and $R \approx R_{\text {hypo }}$.

As a hypothetical exercise, on the left side of Figure 2 we further subdivide streamside exchange into resistors for isotropic turbulence $\left(R_{\text {stream }}^{\mathrm{DSL}}\right)$, coherent turbulence $\left(R_{\text {stream }}^{\mathrm{SRM}}\right)$, and eddies in the cavities between roughness elements $\left(R_{\text {stream }}^{\mathrm{BRM}}\right)$. For the arrangement shown, the total mass transfer resistance for streamside exchange follows from the addition rules for resistors in series and parallel:

$$
R_{\text {stream }}=R_{\text {stream }}^{\text {BRM }}+\left(\frac{1}{R_{\text {stream }}^{\text {DSL }}}+\frac{1}{R_{\text {stream }}^{\text {SRM }}}\right)^{-1}
$$

It should be stressed that the arrangement of mass transfer resistors illustrated in Figure 2 is illustrative only, and will need to be tailored for different environmental flows and mass transport problems. While there is no "one size fits all" solution, the overall approach outlined here is appealing because it provides a simple framework for thinking about, indeed hypothesizing, how different microscale turbulence models might interact to regulate overall interfacial flux at the sediment-water interface.

3: NON-DIMENSIONAL MASS TRANSPORT PARAMETERS: Nondimensional parameters represent the ratio of key time or length-scales that characterize a particular problem. The Sherwood number $S h=\delta /\left(R D_{\mathrm{m}}\right)$ represents the interfacial mass transfer resistance associated with a turbulent flow $(R)$ divided into the (theoretical) mass transfer resistance associated with the molecular diffusion across a characteristic length-scale $\left(\delta / D_{\mathrm{m}}\right)$ where $\delta$ and $D_{\mathrm{m}}$ represent the thickness of the turbulent velocity boundary layer (see Figure 1) and the molecular diffusion coefficient of mass in water, respectively. The Schmidt number Sc = $v / D_{\mathrm{m}}$ represents the relative magnitude of momentum and mass transport by molecular processes, where $v$ is the kinematic viscosity of water and $D_{\mathrm{m}}$ is the molecular diffusion coefficient of mass in water. The shear Reynolds number $\operatorname{Re}_{\tau}=u * \delta / v$ represents the ratio of the largest inertial turbulent scale $(\delta)$ to the smallest dissipative scale in the flow $\left(v / u_{*}\right)$. The Reynolds roughness number $\operatorname{Re}_{\mathrm{k}}=$ $u * k_{\mathrm{s}} / v$ represents the ratio of the bed roughness height $k_{\mathrm{s}}$ to the inner region length-scale $v / u_{*}$. The permeability-based Peclet number $P e_{\mathrm{K}}=u * \sqrt{ } K / D_{\mathrm{m}}^{\prime}$ represents the ratio of shear velocity $\left(u_{*}\right)$ of the flow field and a diffusive velocity in the sediment bed $\left(D_{m}^{\prime} / \sqrt{ } K\right)$.

\section{EMPIRICAL CORRELATIONS FOR MASS TRANSFER RESISTANCE: PRESENT STATE OF AFFAIRS}

Experimental and theoretical studies suggest that all three resistance terms for stream side exchange $\left(R_{\mathrm{stream}}^{\mathrm{BRM}}, R_{\mathrm{stream}}^{\mathrm{DSL}}\right.$ and $R_{\text {stream }}^{\text {SRM }}$ ) can be estimated from the same basic power-law relationship (eq 7), although values of the prefactor $(a=9-50)$ and power-law exponent $(b=1 / 2$ to $2 / 3)$ vary depending on the underlying transport mechanism presumed. ${ }^{20,25-27,43}$

$$
R_{\text {stream }}=a \mathrm{Sc}^{b} / u_{*}
$$

From this last equation, mass transfer resistance caused by streamside exchange can be calculated from the nondimensional Schmidt number (see Box 3) and the ambient turbulence in a stream as represented by the shear velocity, $u_{*}$. Presently, there are no equivalent correlations for $R_{\text {hypo }}$ although in principle one could be calculated from published correlations of the effective diffusion coefficient for hyporheic exchange, $D_{\text {eff }}$ if an appropriate "diffusion length-scale", $L$, was known: $R_{\text {hypo }}=L / D_{\text {eff. }}$ 
Several process-based models have been proposed for the effective diffusion coefficient ${ }^{28,31-33}$ and O'Connor and Harvey ${ }^{38}$ recently developed an empirical correlation that captures much of the variance in hyporheic exchange rates measured over a wide range of flow conditions, sediment bed types (with and without bedforms), and tracer diffusivities:

$$
D_{\text {eff }}=5 \times 10^{-4} \operatorname{Re}_{\mathrm{k}} \mathrm{Pe}_{\mathrm{K}}^{6 / 5} D_{\mathrm{m}}^{\prime}
$$

In this equation, $\mathrm{Re}_{\mathrm{k}}$ and $\mathrm{Pe}_{\mathrm{K}}$ represent the nondimensional Reynolds roughness number and permeability-based Peclet number (see Box 3 ), and $D_{m}^{\prime}=\beta D_{\mathrm{m}}$ is the molecular diffusion coefficient for a tracer in water $D_{\mathrm{m}}$, modified by the constant $\beta$ to account for the twists and turns a molecule experiences as it moves through a porous medium. In the context of the conceptual framework described here the development of a predictive correlation for $R_{\text {hypo }}$ or equivalently the identification of an appropriate physical length-scale for $L$, is an obvious research priority.

\section{EMPIRICAL CORRELATIONS FOR MASS TRANSFER RESISTANCE: LOOKING TO THE FUTURE}

How does insight into the nature of coherent turbulence in boundary layer flows improve our ability to predict values for the mass transfer resistance terms described above? In the fields of chemical and mechanical engineering, turbulent mass transfer to bluff objects is predicted from empirical correlations between nondimensional numbers such as the Sherwood number (Sh), shear Reynolds number $\left(\operatorname{Re}_{\tau}\right)$, and Schmidt number $(\mathrm{Sc})$ (see Box 3): ${ }^{36}$

$$
\mathrm{Sh}=f\left(\operatorname{Re}_{\tau}, \mathrm{Sc}\right)
$$

Equation 9 suggests that the mass transfer resistance in the turbulent boundary layer (as expressed by the value of the Sherwood number) should depend, in general, on both inner (i.e., $u_{*}, v$ ) and outer (i.e., $U_{\infty}, \delta$ ) region variables included in the definitions of the shear Reynolds number and Schmidt number (the shear Reynolds number depends implicitly on the bulk velocity $U_{\infty}$ through the friction coefficient, which relates the bulk velocity to the shear velocity). However, for both published correlations presented in the last section (eqs 7 and 8), the influence of boundary layer turbulence on mass transport is parametrized in terms of only inner region variables $(u *$ and $v$ ). This observation raises a conundrum: if interfacial mass transfer is dominated by sweep and eject events associated with coherent turbulence, $^{25-29}$ and coherent turbulence is modulated by outer variables, ${ }^{17}$ then why does mass transfer across the sedimentwater interface depend only on inner region variables?

The likely answer is that experimental approaches have not historically been designed to identify, or have been capable of identifying, the influence of outer layer region variables on mass transfer at the sediment-water interface. Thus, the effects of the outer region likely manifest as predictive uncertainty or, more concernedly, as uncharacterized bias when results from the laboratory are applied to the field. Such historical constraints are being overcome by dramatic improvements in the spatial resolution and frequency with which fluid velocity and mass concentration fields can be measured near the sediment-water interface, ${ }^{29,44}$ and computing advances that permit computational fluid dynamic simulations of flow paths within stream sediments ${ }^{30,35,42,45}$ and direct numerical solutions of the nonlinear Navier-Stokes equations that govern multiphase flow in the turbulent boundary layer. ${ }^{46}$ These experimental and theoretical advances, together with careful reanalysis of existing data sets, should pave the way for better characterization, and ultimately prediction, of interfacial mass transport in turbulent environmental flows from both inner and outer region variables.

\section{VISION AND PRACTICAL APPLICATIONS}

The experimental and numerical advances described above will likely motivate new mass transfer correlations that include both inner and outer region variables, and thus account for coherent turbulence over a range of Reynolds numbers. These new correlations, when combined with mass transfer circuits like the one shown in Figure 2 can, in principle, predict the flux of a variety of constituents - e.g., oxygen, nutrients, organisms, heavy metals, organic pollutants - across the sediment-water interface in turbulent environmental flows. This vision can be generalized in two important ways. First, mass transfer circuits can be tailored to a variety of environmental settings. As an example, in rivers, coastal waters, and estuaries, resistors can be added in series with both $R_{\text {stream }}$ and $R_{\text {hypo }}$ to account for transport across biogenic roughness (e.g., benthic flora and fauna, such as reeds and corals $^{47-49}$ ) provided that conditions outlined in Box 2 are satisfied. Second, correlations developed for $R_{\text {stream }}$ and $R_{\text {hypo }}$ may prove "universal", in that they apply to many different types of interfacial boundaries (seabed-ocean, sea-air, beach-ocean, land-air, etc.) by appropriate choice of length and velocity scales (e.g., ref 43). If true, this would imply insights obtained from studying mass transport across the turbulent boundary layer in streams, for example, can inform modeling and analysis of interfacial mass transfer in other domains of environmental science and engineering, and vice versa.

\section{AUTHOR INFORMATION}

\section{Corresponding Author}

*E-mail: sbgrant@uci.edu; phone: (949) 824-8277; fax: (949) 824-2541.

\section{BIOGRAPHY}

Stanley Grant is a Professor in the Department of Civil and Environmental Engineering at the University of California Irvine, and a Visiting Chair of Hydrology and Water Resources in the Department of Infrastructure at the University of Melbourne. Ivan Marusic is a Professor and Australian Research Council Federation Fellow in the Department of Mechanical Engineering at the University of Melbourne.

\section{ACKNOWLEDGMENT}

Financial support provided by a 2011 UCI Henry Samueli School of Engineering Research Ignition Fund and a National Science Foundation Award 0724806 (to S.B.G.); and the Australian Research Council and the University of Melbourne School of Engineering Iconic Research Project (to I.M.). We thank M. Stewardson for stimulating conversations and careful review of the manuscript, and the excellent feedback and suggestions of three anonymous reviewers.

\section{REFERENCES}

(1) Pope, S. B. Turbulent Flows; Cambridge University Press: Cambridge, U.K., 2000. 
(2) Thorpe, S. A. The Turbulent Ocean; Cambridge University Press: Cambridge, U.K., 2005.

(3) Cantwell, B. J. Organised motion in turbulent flow. Annu. Rev. Fluid Mech. 1981, 13, 457-515.

(4) Robinson, S. Coherent motions in the turbulent boundary layer. Annu. Rev. Fluid Mech. 1991, 23, 601-639.

(5) Adrian, R. Hairpin vortex organization in wall turbulence. Phys. Fluids 2007, 19, 041301, 1-16.

(6) Marusic, I.; McKeon, B. J.; Monkewitz, P.; Nagib, H. M.; Smits, A. J.; Sreenivasan, K. R. Wall-bounded turbulent flows at high Reynolds numbers: Recent advances and key issues. Phys. Fluids 2010, 22, 065103, 1-24.

(7) Smits, A. J.; McKeon, B. J.; Marusic, I. High Reynolds number wall turbulence. Annu. Rev. Fluid Mech. 2010, 43, 353-375.

(8) Prandtl, L. Uber Flussigkeits bewegung bei sehr kleiner Reibung. In Verhaldlg III Int. Math. Kong.; Teubner: Heidelberg, 1904; pp 484-491.

(9) Nikuradse, J. Gesetzmassigkeiten der turbuleuten Stromung in glatten Rohren. VDI-Forschungsh 1932, 356, 1-36.

(10) Coles, D. E. The law of the wake in the turbulent boundary layer. J. Fluid Mech. 1956, 1, 191-226.

(11) Hutchins, N.; Marusic, I. Evidence of very long meandering streamwise structures in the logarithmic region of turbulent boundary layers. J. Fluid Mech. 2007, 579, 1-28.

(12) Hommema, S. E.; Adrian, R. J. Packet structure of surface eddies in the atmospheric boundary layer. Boundary-Layer Meteorol. 2003, 106, 147-170.

(13) Monty, J.; Stewart, J.; Williams, R.; Chong, M. Large scale features in turbulent pipe and channel flows. J. Fluid Mech. 2007, 589, 147-156.

(14) Schlatter, P.; Örlü, R.; Li, Q.; Brethouwer, G.; Fransson, J. H. M.; Johansson, A. V.; Alfredsson, P. H.; Henningson, D. S. Turbulent boundary layers up to $\mathrm{Re}=2500$ studied through simulation and experiment. Phys. Fluids 2009, 21, 051702, 1-4.

(15) Marusic, I. Unraveling near wall turbulence. J. Fluid Mech. 2009, 632, 431-442.

(16) Jimenez, J. Recent developments on wall-bounded turbulence. Rev. R. Acad. Cien. A 2007, 101 (2), 187-203.

(17) Marusic, I.; Mathis, R.; Hutchins, N. Predictive model for wallbounded turbulent flow. Science 2010, 329, 193-196.

(18) Hester, E. T.; Gooseff, M. N. Moving beyond the banks: Hyporheic restoration is fundamental to restoring ecological services and functions of streams. Environ. Sci. Technol. 2010, 44, $1521-1525$.

(19) The Hyporheic Handbook: A Handbook on the GroundwaterSurface Water Interface and Hyporheic Zone for Environment Managers; Integrated Catchment Science Programme, Science report SC040070; Environment Agency: Bristol, U.K., 2010.

(20) Dade, W. B. Near-bed turbulence and hydrodynamic control of diffusional mass transfer at the sea floor. Limnol. Oceanogr. 1993, 38 (1), 52-69.

(21) Ren, J.; Packman, A. I. Stream-subsurface exchange of Zinc in the presence of Silica and Kaolinite colloids. Environ. Sci. Technol. 2004, 38, 6571-6581.

(22) Grant, S. B.; Litton-Mueller, R. M.; Ahn, J. H. Measuring and modeling the flux of fecal bacteria across the sediment-water interface in a turbulent stream, Water Resour. Res. 2011, 47, W05517, doi:10.1029/ 2010WR009460.

(23) Grant, S. B.; Sanders, B. F. Beach Boundary Layer: A framework for addressing recreational water quality impairment at enclosed beaches. Environ. Sci. Technol. 2010, 44, 8804-8813, DOI: 10.1021/es101732m.

(24) Searcy, K. E.; Packman, A. I.; Atwill, E. R.; Harter, T. Deposition of Cryptosporidium Oocysts in streambeds. Appl. Environ. Microbiol. 2006, 72 (3), 1810-1816.

(25) O'Connor, B. L.; Hondzo, M. Dissolved oxygen transfer to sediments by sweep and eject motions in aquatic environments. Limnol. Oceangr. 2008, 53 (2), 566-578.

(26) Dipprey, D. F.; Sabersky., R. H. Heat and momentum transfer in smooth and rough tubes at various Prandtl numbers. Int. J. Heat Mass Transfer 1963, 6, 329-353.
(27) Inoue, T; Nakamura, Y. Effects of hydrodynamic conditions on DO transfer at a rough sediment surface. ASCE J. Environ. Eng. 2011, 137 (1), 28-37.

(28) Elliott, A. H.; Brooks, N. H. Transfer of nonsorbing solutes to a streambed with bed forms: Laboratory experiments. Water Resour. Res. 1997, 33 (1), 137-151.

(29) Reidenbach, A.; Limm, M.; Hondzo, M.; Stacey, M. T. Effects of bed roughness on boundary layer mixing and mass flux across the sediment-water interface. Water Resour. Res. 2010, 46, doi:10.1029/ 2009WR008248.

(30) Tonina, D.; Buffington, J. M. Hyporheic exchange in gravel bed rivers with pool-riffle morphology: laboratory experiments and threedimensional modeling. Water Resour. Res. 2007, 43, doi: 10.1029/ 2005 WR004328.

(31) Fries, S. Predicting interfacial diffusion coefficients for fluxes across the sediment-water interface. ASCE J. Hydraulic Eng. 2007, 133 (3), 267-272.

(32) Packman, A. I.; Salehin, M.; Zaramella, M. Hyporheic exchange with gravel beds: Basic hydrodynamic interactions and bedform-induced advective flows. ASCE J. Hydraulic Eng. 2004, 130 (7), 647-656.

(33) Higashino, M.; Clark, J. J. ; Stefan. H. G. Pore water flow due to near-bed turbulence and associated solute transfer in a stream or lake sediment bed. Wat. Resour. Res. 2009, 45, doi: 10.1029/2008WR007374.

(34) Worman, A.; Packman, A. I.; Johansson, H.; Jonsson, K. Effect of flow-induced exchange in hyporheic zones on longitudinal transport of solutes in streams and rivers. Water Resour. Res. 2002, 38 (1), doi: 10.1029/2001WR000769.

(35) Stonedahl, S. H.; Harvey, J. W. ; Worman, A. ; Salehin, M.; Packman, A. I. A multiscale model for integrating hyporheic exchange from ripples to meanders. Water Resources Res. 2010, 46, doi:10.1029/ 2009WR008865.

(36) Incropera, F. P., Dewitt, D. P., Bergman, T. L., Lavine, A. S. Fundamentals of Heat and Mass Transfer: John Wiley and Sons: Hoboken, NJ, 2007.

(37) Valasaraj, K. T.; Thibodeaux, L. T.; Reible, D. D. A quasisteady-state pollutant flux methodology for determining sediment quality criteria. Environ. Toxicol. Chem. 1997, 16 (3), 391-396.

(38) O'Connor, B. L.; Harvey, J. W. Scaling hyporheic exchange and its influence on biogeochemical reactions in aquatic ecosystems. Water Resour. Res. 2008, 44, doi:10.1029/2008WR007160.

(39) Higashino, M.; Stefan, H. G. Near-bed turbulence models: Significance for diffusional mass transfer at the sediment/water interface. J. Hydraulic Res. 2008, 46 (3), doi:10.3826/jhr.2008.2769.

(40) Bencala, K. E.; Gooseff, M. N.; Kimball, B. A. Rethinking hyporheic flow and transient storage to advance understanding of stream-catchment connections. Water Resour. Res. 2011, 47, W00H03, doi:10.1029/2010WR010066.

(41) Basagaoglu, H.; Ginn, T. R.; Green, C. T.; McCoy, B. J. Transport in heterogeneous media: Tracer dynamics in complex flow networks. AIChE J. 2002, 48 (5), 1121-1131.

(42) Storey, R. G., Howard, K. W. F., Williams, D. D. Factors controlling riffle-scale hyporheic exchange flows and their seasonal changes in a gaining stream: A three dimensional groundwater flow model. Water Resour. Res. 2003, 39, doi:10.1029/2002WR001367.

(43) Lorke, A.; Peeters, F. Toward a unified scaling relation for interfacial fluxes. J. Phys. Oceanogr. 2006, 36, 955-961.

(44) Lorrai, C.; McGinnis, D. F.; Berg, P.; Brand, A.; Wuest, A. Application of oxygen eddy correlation in aquatic systems. J. Atmos. Oceanic Technol. 2010, 27, 1533-1546.

(45) Boano, F.; Revelli, R.; Ridolfi, L. Water and solute exchange through flat streambeds induced by large turbulent eddies. J. Hydrol. 2011, 402, 290-296.

(46) Ahmed, A. M.; Elghobashi, S. Direct numerical simulation of particle dispersion in homogeneous turbulent shear flows. Phys. Fluids. 2001, 13 (11), 3346-3364.

(47) Stoesser, T.; Kim, S. J.; Diplas, P. Turbulent flow through idealized emergent vegetation. ASCE J. Hydraul. Eng. 2010, 136 (12), 1003-1017, DOI: 10.1061/(ASCE)HY.1943-7900.0000153. 
(48) Ghisalberti, M.; Nepf, H. Shallow flows over a permeable medium: The hydrodynamics of submerged aquatic canopies. Transp. Porous Media 2009, 78 (3), 385-402, DOI: 10.1007/s11242-00909434-x.

(49) Monismith, S. G.; Davis, K. A.; et al. Flow effects on benthic grazing on phytoplankton by a Caribbean Reef. Limnol. Oceanogr. 2010, 55 (5), 1881-1892, DOI: 10.4319/lo.2010.55.5.1881.

(50) Okamoto, N.; Yoshimatsu, K.; Schneider, K.; Farge, M.; Kaneda, Y. Coherent vortices in high resolution direct numerical simulation of homogeneous isotropic turbulence: A wavelet viewpoint. Phys. Fluids 2007, 19, 115109, 1-14.

(51) Ganapathisubramani, B.; Longmire, E. K.; Marusic, I. Experimental investigation of vortex properties in a turbulent boundary layer. Phys. Fluids 2006, 18, 055105, 1-15. 